\title{
Low-Density Lipoprotein (LDL) Antioxidants and Antiplatelet Compounds from Selected Malaysian Plants as Leads for the Development of Cardiovascular Protective Agents
}

\author{
Ibrahim Jantan
}

Drug and Herbal Research Center, Faculty of Pharmacy, Universiti Kebangsaan Malaysia, Jalan Raja Muda Abdul Aziz, 50300 Kuala Lumpur, Malaysia; E-mail: ibj@pharmacy.ukm.my

Oxidative modification of low-density lipoprotein (LDL) plays a significant role in the onset and acceleration of atherosclerotic process and the development of cardiovascular diseases. The chronic antioxidant, hypolipidemic, antithrombotic and antiplatelet activities of many phytochemicals have important roles in prevention of lipoprotein oxidation and atherosclerotic lesion development. Phytochemicals of diverse chemical structures, including phenolic compounds, xanthones, prenylflavonoids, benzophenones, alkaloids and triterpenoids isolated from Malaysian plants were investigated for their ability to inhibit arachidonic acid (AA), collagen and adenosine diphosphate (ADP) induced platelet aggregation in human whole blood using an electrical impedance method. The compounds were also investigated for their ability to inhibit human LDL oxidation using thiobarbituric acid reactive substances (TBARS) assay. Among the compounds tested, 2-(3-methylbut-2-enyl)1,3,5,-trihydroxyxanthone, 2-(3-methylbut-2-enyl)-1,3,5,6-tetrahydroxyxanthone, rubraxanthone and 3,5,3',5'-tetrahydroxy-4methoxybenzophenone isolated from Guttiferae species showed strong inhibition on platelet aggregation. Curcumin, cardamonin, pinocembrine, 5,6-dehydrokawain and 3-deacetylcrotepoxide were the strong antiplatelet compounds identified from Zingiberaceae species. Of the alkaloids tested, (-)-N-acetylnornuciferine, (-)-N-acetylanonaine and 1-(N-acetyl-Nmethylamino)ethyl-3,4,6-trimethoxy-7-hydroxyphenanthrene from Armodendron elegans showed strong inhibition on platelet aggregation caused by all three inducers. 3,5,3',5'-Tetrahydroxy-4-methoxybenzophenone and 1, 7-dihydroxyxanthone showed strong inhibitory activity on LDL oxidation. Curcumin, demethoxycurcumin and bisdemethoxycurcumin exhibited stronger activity than the reference, probucol, as LDL antioxidants. Xanthorrhizol, zerumbone and ar-turmerone from the oils of Curcuma species showed relatively strong antioxidant activity. 3,5,3',5'-Tetrahydroxy-4-methoxybenzophenone and 1, 7dihydroxyxanthone also showed strong inhibitory activity on LDL oxidation. Of the triterpenoids tested, garcihombronane B from Garcinia cantleyana showed moderate activity against LDL oxidation. The compounds are new classes of natural products that can be used as leads for development of new cardiovascular protective agents.

Keywords: Xanthones, prenylflavonoids, benzophenones, curcumin, antiplatelet aggregation, low-density lipoprotein antioxidants. 